\title{
Modelo de gestión de riesgo; su influencia con la gestión de calidad en el sector plástico en la ciudad de Bogotá
}

\author{
pags $36-49$ \\ Grupo de Investigación: Centro de Investigación y Desarrollo Empresarial CINDE \\ Línea de investigación: Desarrollo Mipymes \\ Carlos Gabriel Correa Ch.• \& Luis Miguel Enciso Piza»
}

Recibido: 5 de julio de 2015

Aceptado: 5 de septiembre de 2015

\begin{abstract}
RESUMEN
El presente trabajo muestra un paso a paso de un modelo de gestión de riesgos bajo la norma NTC ISO $31000: 2009$, la cual se despliega como una herramienta administrativa que facilita la toma de decisiones que puede verse afectadas por la incertidumbre y poder proteger el valor en todas las actividades que afectan a la organización. Se hizo una prueba piloto aplicando los conceptos y teorias necesarias para desarrollarlo y se realizó el proceso de la gestión de riesgo donde se identifican, evalúan y analizan los riesgos identificados, y su respectivo tratamiento para reducir el impacto del riesgo.
\end{abstract}

Palabras clave: gestión de riesgo, beneficios, valores, tratamiento del riesgo, incertidumbre.

\begin{abstract}
This paper shows a step of a risk management model under the NTC ISO 31000: 2009, which is deployed as a management tool that facilitates decision-making that may be affected by uncertainty and to protect the value in all activities that affect the organization. A pilot was applying the concepts and theories needed to develop and the process of risk management which identifies, evaluates and analyzes the risks identified, and their respective treatment is performed to reduce the impact of risk.
\end{abstract}

Keywords: risk management, benefits, values, risk Treatment, uncertainty.

\footnotetext{
- Docente investigador Universidad de América, Programa: Ingeniería industrial. carlos.correa@investigadores.uamerica.edu.co Ingeniero Industrial 1977

•• Estudiante de Décimo semestre de Ingeniería Industrial. CO-investigador CINDE, en la línea Desarrollo Mipymes, semillero de Investigación del grupo CINDE.
} 


\section{INTRODUCCIÓN}

Las empresas de todos los tamaños y diversas actividades económicas buscan alcanzar el logro de sus objetivos empresariales, los cuales se pueden variar como metas propuestas por la alta gerencia o el cumplimiento de los requisitos legales y reglamentarios con entidades externas a ellas, sin embargo, los cambios en el entorno donde debe interactuar la organización, generan incertidumbre ya sea por el desconocimiento del acontecimiento o por el grado de decisiones que debe tomar para el desarrollo de la estrategia, generando una desviación en lo que se espera tanto positiva como negativa o ambos. Para este caso se estudian las consecuencias de generan impacto negativo. La desviación con consecuencias negativas en el resultado esperado se conoce como riesgo.

Las empresas Pymes productoras de empaques y envases plásticos que exportan son conscientes de identificar y tratar los riesgos asociados a sus actividades económicas, ya que al exportar se asumen riesgos de pérdidas en la inversión y de participación de sus mercados. Desarrollar un modelo de gestión de riesgo les permite dar un proceso que gestione el riesgo y que les facilite tomar decisiones con respecto a los riesgos asociados en su actividad económica.

La gestión de riesgos esta estandarizada según ICONTEC con la norma NTC ISO 31000:2009, la cual ofrece principios y directrices para implementar una gestión de riesgos, pero no ofrece una base para que las organizaciones la implementen de manera eficaz. Por lo tanto, se desarrolla el modelo de gestión de riesgo y este se influencia en la gestión de calidad ya que esta ofrece una base confiable y de información en los procesos de la empresa para que el proceso de gestión de riesgo se desarrolle de manera eficaz y sea muy sencillo su ejecución, lo que permite generar los beneficios esperados por este procedimiento.

El trabajo de investigación realizó prueba piloto del modelo de gestión de riesgo en la empresa ELASTOPLAST Ltda., elegida entre las empresas que exportan y son productoras de envases y empaques plásticos en la ciudad de Bogotá, donde se segmento el tamaño de la muestra por una base de datos adquirida en la Cámara de Comercio de Bogotá.

\section{MÉTODO}

El desarrollo del trabajo de investigación planteó el paso a paso del modelo de gestión de riesgo (Ver Diagrama 1), el cual fue diseñado en cuatro fases: a. dirección y compromiso junto a los requerimientos y beneficios en el proceso de gestión de riesgos, b. análisis de contexto que permite desarrollar el marco de referencia, c. el proceso para la gestión del riesgo y d. el tratamiento del riesgo junto con el monitoreo y revisión.

\subsection{Dirección y compromiso}

La base determinante para el modelo de gestión de riesgo parte del compromiso que se presente por parte de la alta gerencia. La dirección y compromiso se divide en los principios tanto de la gestión de riesgo como los parámetros para aplicarse en las personas en la organización, se continúa con el compromiso en la dirección y se finaliza con los beneficios esperados por la gestión de riesgos.

\subsubsection{Principios}

Los principios son pilares de políticas para el logro misional en todas las organizaciones, de ahí forman el carácter en los trabajadores y la cultura organizacional. La norma NTC ISO 31000:2009 establece los principios de la gestión de riesgos como se muestra en la Tabla 1, los cuales aplicados con los valores de la organización forma una base para que la gestión de riesgos sea eficaz.

Según Juan Carlos Jiménez (El valor de los valores en las organizaciones. 2010), Los valores se expresan en la toma de decisiones de las per- 


\section{Diagrama 1. Flujograma modelo de gestión de riesgos}

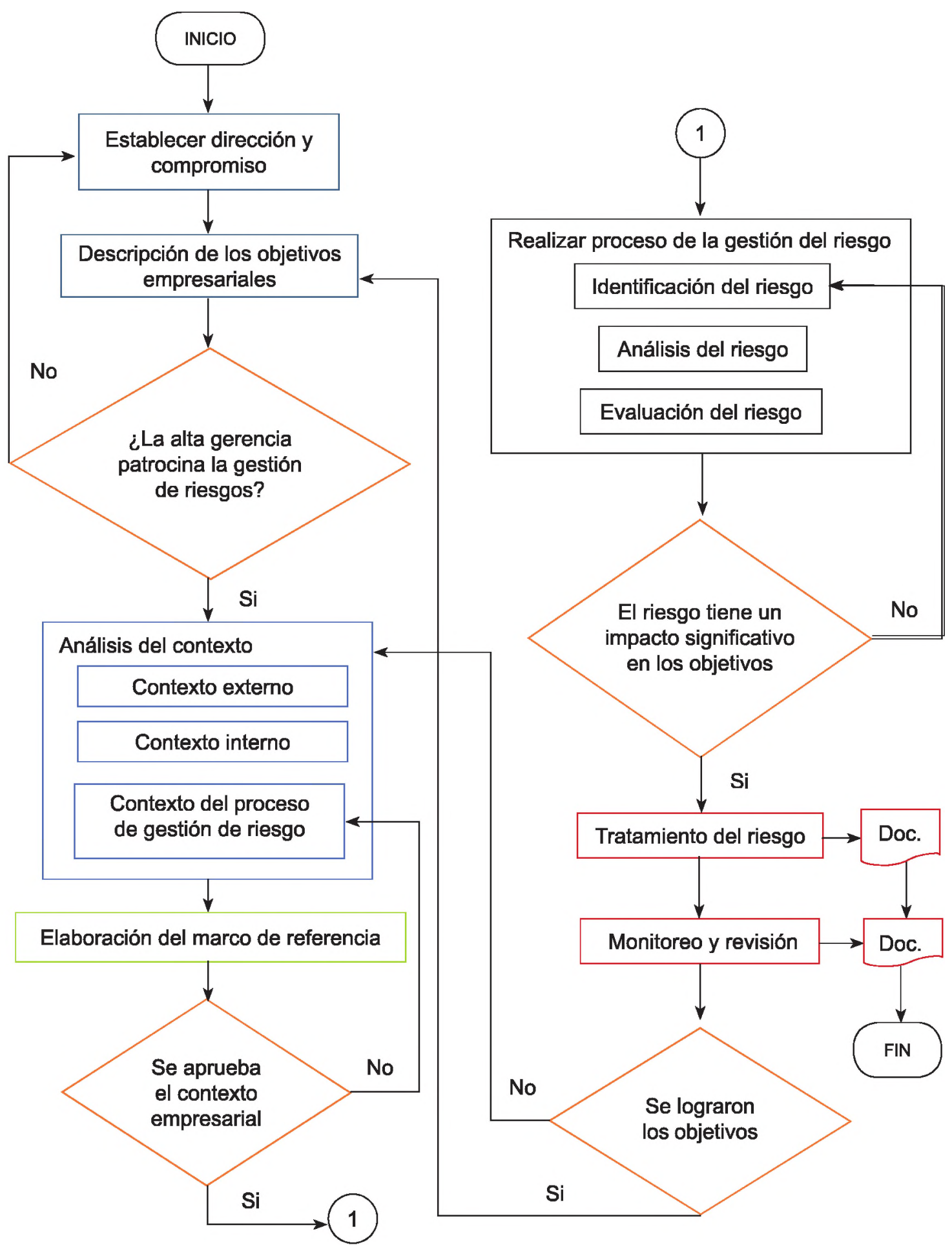

Fuente: los autores. 
LÍNEA DE INVESTIGACIÓN: DESARROLLO MIPYMES

sonas y son éstas, junto a sus prácticas y talentos, lo que hace posible la entrega de valor a los clientes. Los valores en las organizaciones necesitan tener sentido práctico de la siguiente manera.

- Que todos los miembros las conozcan.

- Que todos estén de acuerdo con su significado.

- Que todos comprendan los comportamientos que implican.

- Que todos los pongan en práctica.

Cuando las personas de una organización "trabajan los valores que los unen, logran sus objetivos de manera más eficiente y satisfactoria." Sus integrantes se sienten más unidos y motivados a formar parte de ella, pero ¿por qué se debilitan los valores? Los valores se debilitan básicamente por tres razones.

Las necesidades pueden presionar más que los valores. Los valores no se deterioran. Lo que en realidad se debilita es la propia capacidad de creer en determinados principios y su pertinencia, como producto de la presión que ejerce sobre el manejo de ciertas necesidades.

Es mucho más fácil transmitir otros valores. El verdadero significado de los valores en las organizaciones se basa en las actitudes de sus integrantes, y el comportamiento se traduce en detalles de lo que se hace en el día a día.

Existe mucha presión social a favor de "antivalores". En una sociedad que sobre-estimula el consumo y la propiedad, los ciudadanos terminan siendo valorados más por lo que son como personas. En consecuencia, la apariencia o el poder muchas veces se convierten en valores superiores a la responsabilidad.

Según la Norma NTC ISO 31000 para garantizar su eficacia continua, se requiere de un compromiso fuerte y sostenido por parte de la dirección de la organización, así como de planificación estratégica y rigurosa para lograr el compromiso a todo nivel.:

\subsubsection{Compromiso}

Según la norma NTC ISO 31000:2009 (ICONTEC), propone los siguientes requisitos para la gestión de riesgos los cuales son: definir y aprobar la política para la gestión del riesgo; garantizar que la cultura de la organización y la política para la gestión del riesgo están alineadas; determinar indicadores del desempeño de la gestión para el riesgo que estén acordes con los indicadores del desempeño de la organización; alinear los objetivos de la gestión del riesgo con los objetivos y las estrategias de la organización; garantizar la conformidad legal y reglamentaria; asignar obligaciones y responsabilidades en los niveles respectivos dentro de la organización; y garantizar que se asignan los recursos necesarios para la gestión del riesgo; comunicar los beneficios de la gestión del riesgo a todas las partes involucradas.

\subsubsection{Beneficios}

La Norma NTC ISO 31000 da a conocer los beneficios al implementar la gestión del riesgo en la organización, para facilitar el análisis según Mario Gutiérrez (2009, pp. 246-247) se hizo un diagrama de árbol, como se muestra en el Diagrama 2.

El principal beneficio es el aumento de la probabilidad de alcanzar los objetivos, se expresa como el efecto que busca la organización al implementar esta norma contrarrestando la incertidumbre que genera el riesgo.

\subsection{Análisis de contexto}

Variables macroeconómicas más relevantes son la balanza comercial, ya que el aumento de las importaciones afecta la participación del mercado de las empresas nacionales, por lo que deben de asumir el riesgo de explorar en mercados internacionales. Otro factor fundamental en el contexto externo es identificar cuáles son los requisitos legales y reglamentarios con las partes involucradas. 
Tabla 1. Principios NTC ISO 31000:2009

\begin{tabular}{|c|c|}
\hline Principio. & Descripción \\
\hline Crear y proteger el valor. & $\begin{array}{l}\text { Contribuye al logro demostrable de los objetivos y a la mejora del desem- } \\
\text { peño en las diferentes áreas de la empresa. }\end{array}$ \\
\hline $\begin{array}{l}\text { Es una parte integral de todos } \\
\text { los procesos de la organización. }\end{array}$ & $\begin{array}{l}\text { No es una actividad independiente que se separa de las actividades y los } \\
\text { procesos principales de la organización. }\end{array}$ \\
\hline $\begin{array}{l}\text { Es parte de la toma de decisio- } \\
\text { nes }\end{array}$ & $\begin{array}{l}\text { Ayuda a quienes toman las decisiones a hacer elecciones informadas, } \\
\text { priorizar acciones y distinguir entre cursos de acción alternativos. }\end{array}$ \\
\hline $\begin{array}{l}\text { Aborda explícitamente la incer- } \\
\text { tidumbre }\end{array}$ & $\begin{array}{l}\text { La gestión del riesgo toma en consideración explícitamente a la incerti- } \\
\text { dumbre, su naturaleza y la forma en que se puede tratar. }\end{array}$ \\
\hline $\begin{array}{l}\text { Es sistemática, estructurada y } \\
\text { oportuna }\end{array}$ & $\begin{array}{l}\text { Un enfoque sistemático, oportuno y estructurado para la gestión del ries- } \\
\text { go contribuye a la eficiencia y a resultados consistentes, comparables y } \\
\text { confiables. }\end{array}$ \\
\hline $\begin{array}{l}\text { Se basa en la mejor informa- } \\
\text { ción disponible. }\end{array}$ & $\begin{array}{l}\text { Las entradas para el proceso de gestión de riesgo se basan en fuentes de } \\
\text { información tales como datos históricos, experiencia, retroalimentación de } \\
\text { las partes involucradas, observación, previsiones y examen de expertos. }\end{array}$ \\
\hline Está adaptada. & $\begin{array}{l}\text { Se alinea del contexto externo e interno y del perfil de riesgo de la organi- } \\
\text { zación. }\end{array}$ \\
\hline $\begin{array}{l}\text { Toma en consideración los fac- } \\
\text { tores humanos y culturales. }\end{array}$ & $\begin{array}{l}\text { Reconoce las capacidades, percentiles e intenciones de individuos } \\
\text { externos e internos, los cuales pueden facilitar o dificultar el logro de los } \\
\text { objetivos de la organización. }\end{array}$ \\
\hline Es transparente e inclusiva. & $\begin{array}{l}\text { La correcta y oportuna intervención de las partes involucradas y, en } \\
\text { particular, de aquellos que toman las decisiones en todos los niveles de la } \\
\text { organización, garantiza que la gestión del riesgo siga siendo pertinente y } \\
\text { se actualice. }\end{array}$ \\
\hline $\begin{array}{l}\text { Es dinámica, reiterativa y recep- } \\
\text { tiva al cambio. }\end{array}$ & $\begin{array}{l}\text { Siente y responde continuamente al cambio. A medida que se presentan } \\
\text { los eventos externos e internos, el contexto y el conocimiento cambian, } \\
\text { tienen lugar el monitoreo y la revisión de los riesgos, emergen riesgos } \\
\text { nuevos, algunos cambian y otros desaparecen. }\end{array}$ \\
\hline $\begin{array}{l}\text { Facilita la mejora continua de la } \\
\text { organización. }\end{array}$ & $\begin{array}{l}\text { Las organizaciones deberían desarrollar e implementar estrategias para } \\
\text { mejorar la madurez de su gestión de riesgos junto con otros aspectos de } \\
\text { su organización. }\end{array}$ \\
\hline
\end{tabular}

Fuente: ICONTEC. Norma NTC ISO 31000:2009. Bogotá

D.C. 2011. 9-11 p. 


\subsection{Justificación y delimitación}

La justificación se dio el concepto de porque se puede implementar la gestión de riesgo (ver Tabla 2), y la delimitación explica los criterios asociados a los recursos a utilizar (ver Tabla 3) y el nivel de integración en las áreas de la empresa (ver Tabla 4). Al delimitar la gestión de riesgo permitió segmentar el proceso ya que a mayor integración entre los departamentos im- plementados en la gestión del riesgo, mayores deben ser los recursos necesarios para su funcionamiento.

Cada criterio presenta una escala de calificación alta, media y baja; y en la Tabla 2, se presenta la calificación del cruce de variables entre los criterios (ver Tabla 3 y Tabla 4) y las diferentes alternativas en la implementación de la gestión de riesgos.

\section{Diagrama 2. Beneficios de la implementación de la gestión de riesgos}

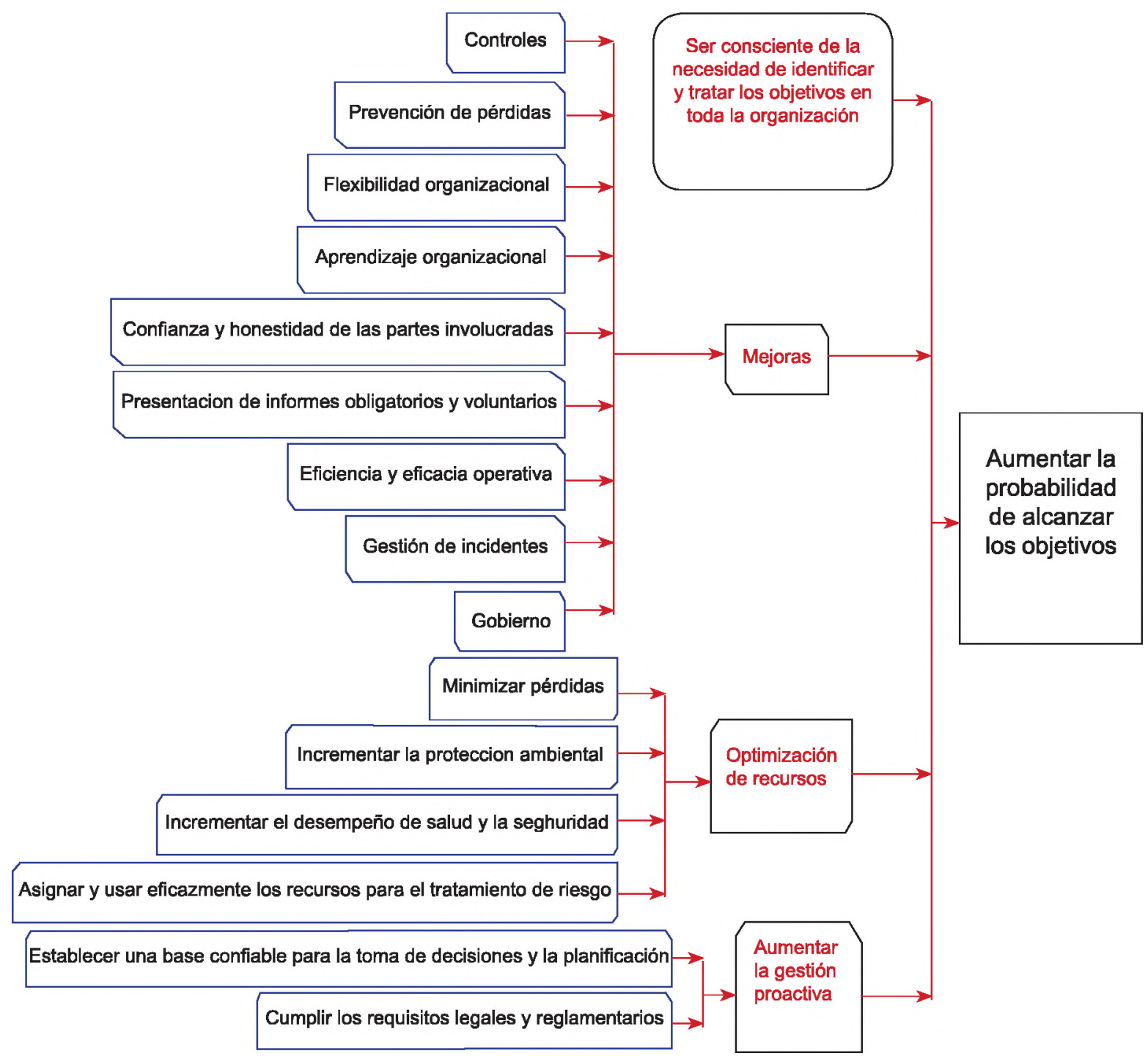

Fuente: Elaborado y modificado por el autor basado en ICONTEC. Norma NTC ISO 31000. Bogotá D.C. 2009. 2 p 
Gráfica 1. Balanza comercial 2013-2014 fabricación de productos plásticos

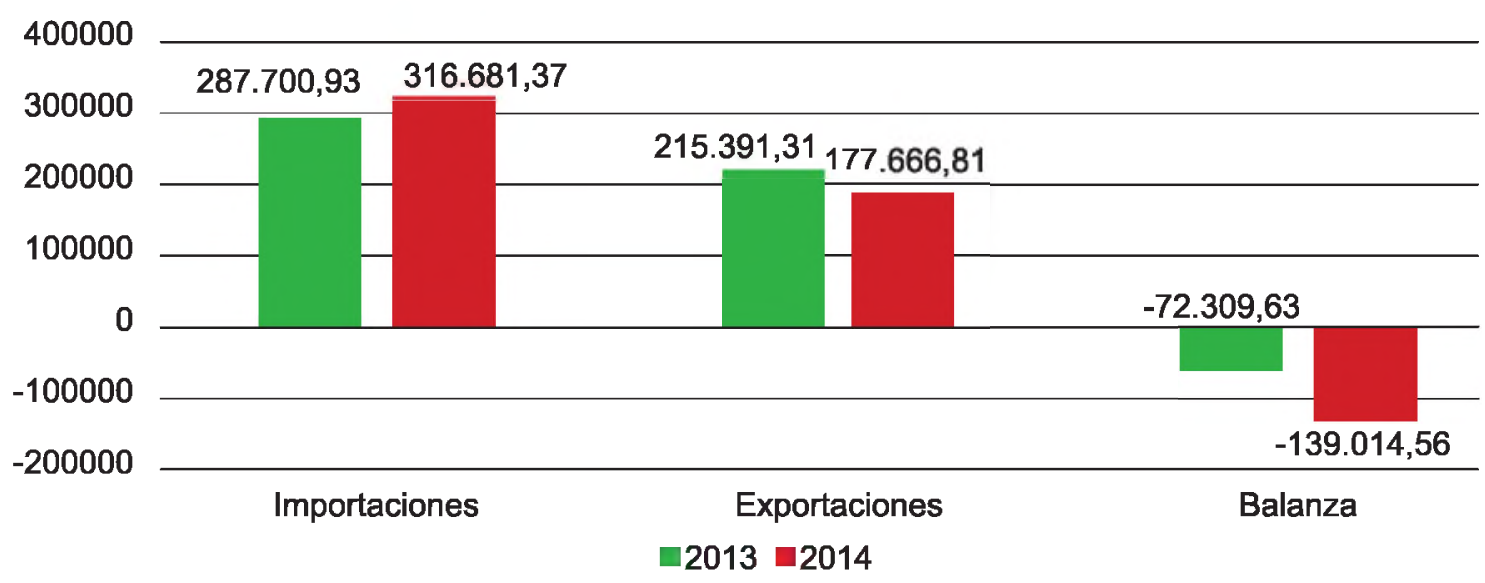

Fuente: DANE (2014)

Tabla 2. Alternativas en la implementación del Sistema de Gestión de Riesgos

\begin{tabular}{|l|l|l|l|}
\hline \multicolumn{2}{|c|}{ Alternativa } & \multicolumn{2}{|c|}{$\begin{array}{c}\text { Nivel de integración } \\
\text { entre las áreas de } \\
\text { apoyo }\end{array}$} \\
\hline $\begin{array}{l}\text { A la par con otros Siste- } \\
\text { mas de gestión }\end{array}$ & $\begin{array}{l}\text { Sistemas de gestión ya implementar } \\
\text { o en proceso. (Ejemplos: ISO 9001, } \\
\text { ISO 14001, ISO 18001, ISO 27001, ISO } \\
\text { 26000, entre otros) }\end{array}$ & Medio & Medio \\
\hline Prueba piloto & $\begin{array}{l}\text { Se hace con el fin de aprender al } \\
\text { máximo para mejorar falencias, con el } \\
\text { propósito de su implementación en toda } \\
\text { la empresa o área específica. }\end{array}$ & Bajo & Bajo \\
\hline Unidad(es) de negocio & $\begin{array}{l}\text { Se puede iniciar por el área más avan- } \\
\text { zada en el tema o de mayor control. }\end{array}$ & Medio & Medio \\
\hline Procesos & $\begin{array}{l}\text { Normativos, básicos de apoyo y de } \\
\text { mejora continua de acuerdo al enfoque } \\
\text { basado en procesos }\end{array}$ & Bajo & Bajo \\
\hline Un riesgo en particular & $\begin{array}{l}\text { Aquel en el que se tena la mayor nece- } \\
\text { sidad, como por ejemplo salud, medio } \\
\text { ambiente, entre otros. }\end{array}$ & Bajo & Medio \\
\hline Toda la empresa & $\begin{array}{l}\text { La integración de todos los departa- } \\
\text { mentos de la empresa en la gestión de } \\
\text { riesgos. }\end{array}$ & Alto & Alto \\
\hline
\end{tabular}

Fuente: Elaborado y modificado por el autor basado en BRAVO Oscar, SÁNCHEZ Marleny. 2012. 
LÍNEA DE INVESTIGACIÓN: DESARROLLO MIPYMES

Las diferentes alternativas presentadas en la Tabla 2, pueden ayudar a que la organización realice un plan de acción que integre todos sus departamentos o si lo prefiere, en las áreas que mayores riesgos se puedan presentar.
La empresa ELASTOPLAST Ltda., delimitó la gestión de riesgo como una prueba piloto enfocada en el logro de sus objetivos de calidad, con el fin de ir adaptando un sistema de gestión de riesgos que fortalezca la gestión de calidad.

Tabla 3. Criterio de evaluación 1

Recursos a utilizar

Criterio de evaluación

Medida

\begin{tabular}{|l|l|}
\hline Alto & Presupuesto entre 50 al $20 \%$ de los ingresos \\
\hline Medio & Presupuesto entre 19 al $8 \%$ de los ingresos \\
\hline Bajo & Presupuesto menos del $8 \%$ de los ingresos \\
\hline
\end{tabular}

Fuente: El autor.

Tabla 4. Criterio de evaluación 2

\begin{tabular}{|c|c|}
\hline & Nivel de integración entre las áreas de apoyo \\
\hline Criterio de evaluación & Medida \\
\hline Alto & $\begin{array}{l}\text { Entre un } 100 \text { a } 80 \% \text { de los departamentos o áreas y partes interesadas de la } \\
\text { organización }\end{array}$ \\
\hline Medio & $\begin{array}{l}\text { Entre un } 79 \text { a } 40 \% \text { de los departamentos o áreas y partes interesadas de la } \\
\text { organización }\end{array}$ \\
\hline Bajo & $\begin{array}{l}\text { Entre } 39 \text { a } 1 \% \text { de los departamentos o áreas y partes interesadas de la orga- } \\
\text { nización }\end{array}$ \\
\hline
\end{tabular}

Fuente. El autor.

\subsection{Proceso de la gestión del riesgo}

Una vez determinado el contexto, alcance y justificación del modelo, el proceso para la gestión de riesgo se divide en: a) identificación de riesgo, b) análisis del riesgo y c) evaluación del riesgo.

\subsubsection{Identificación del riesgo}

La identificación de los riesgos se plantea en la Tabla 5, la cual surgió de una lluvia de ideas, donde el equipo de trabajo para la gestión de riesgos consideró que pueden afectar en los objetivos empresariales.

En la identificación del riesgo se busca responder tres preguntas: ¿qué puede suceder?
¿Por qué puede suceder?, ¿cuáles son las consecuencias?, las cuales explican las causas los efectos y cuál es el evento de riesgo.

\subsubsection{Análisis del riesgo}

Una vez identificados los posibles riesgos que afectan el cumplimiento de los objetivos empresariales, la empresa determinó la manera de calcular la probabilidad y la consecuencia, variables necesarias para evaluar y valorar los riesgos. Se determina la calificación de la probabilidad en la Tabla 6 y la consecuencia de acuerdo con la Tabla 7 y Tabla 8 , para el cálculo de la probabilidad la empresa se basó en datos históricos obtenidos en su sistema de gestión de calidad. Con respecto a la forma de evaluar la consecuencia, se deter- 


\begin{tabular}{|c|c|c|c|}
\hline Riesgo & ¿Qué puede suceder? & ¿Por qué puede suceder? & ¿Cuáles son las consecuencias? \\
\hline $\begin{array}{l}\text { Defectos en los produc- } \\
\text { tos generados en su } \\
\text { transporte. }\end{array}$ & \begin{tabular}{|l|} 
Dependiendo del modo que se \\
transporte la mercancía, ya sea \\
por tierra mar o aire, se pueda \\
perjudicar por diversas perturba- \\
ciones en su recorrido.
\end{tabular} & $\begin{array}{l}\text { - La empresa ofrece un pésimo servicio } \\
\text { y tiene poco cuidado con la mercancía } \\
\text { embalada. } \\
\text { - Mala distribución del embalaje. } \\
\text { - Poca especificación y cuidados nece- } \\
\text { sarios para que envío este en óptimas } \\
\text { condiciones. }\end{array}$ & $\begin{array}{l}\text { Descontento por parte de los } \\
\text { clientes. } \\
\text { Pago de multas por mal estado } \\
\text { de la mercancía. }\end{array}$ \\
\hline $\begin{array}{l}\text { Cantidad errónea de } \\
\text { productos entregados. }\end{array}$ & $\begin{array}{l}\text { Se presenta cuando se envía } \\
\text { menor o mayor cantidad de mer- } \\
\text { cancía que se tenía acordada } \\
\text { por las partes. }\end{array}$ & $\begin{array}{l}\text { - Errores en la cantidad a enviar. } \\
\text { Poco inventario para satisfacer el pedi- } \\
\text { do. } \\
\text { La producción no fue la suficiente para } \\
\text { satisfacer el pedido. }\end{array}$ & $\begin{array}{l}\text { - Sobrecostos del pedido, por lo } \\
\text { que se debe hacer sostenimiento } \\
\text { de acuerdo a los requerimientos } \\
\text { especificados por los clientes. } \\
\text { - Desmotivación de los clientes. } \\
\text { - Pago de multas por incumpli- } \\
\text { miento. }\end{array}$ \\
\hline $\begin{array}{l}\text { Pérdida de la imagen } \\
\text { corporativa. }\end{array}$ & $\begin{array}{l}\text { La empresa se da cuenta que la } \\
\text { opinión de sus clientes es muy } \\
\text { mala con respecto a los produc- } \\
\text { tos que ofrece }\end{array}$ & $\begin{array}{l}\text { Errores en la entrega, mal estado del } \\
\text { producto o insatisfacción de las necesi- } \\
\text { dades de uno o varios clientes. } \\
\text { Cambios de directivos que imponen nue- } \\
\text { vos principios que van en contra de los } \\
\text { valores tenían establecidos. }\end{array}$ & $\begin{array}{l}\text { Pérdida de participación en el } \\
\text { mercado donde venden los pro- } \\
\text { ductos. } \\
\text { Desprestigio por parte de los } \\
\text { clientes que toman la decisión } \\
\text { de no comprarle sus productos } \\
\text { porque están disgustados. }\end{array}$ \\
\hline $\begin{array}{l}\text { Pérdida de la imagen } \\
\text { corporativa. }\end{array}$ & $\begin{array}{l}\text { La empresa se da cuenta que la } \\
\text { opinión de sus clientes es muy } \\
\text { mala con respecto a los produc- } \\
\text { tos que ofrece }\end{array}$ & $\begin{array}{l}\text { Errores en la entrega, mal estado del } \\
\text { producto o insatisfacción de las necesi- } \\
\text { dades de uno o varios clientes. } \\
\text { cambios de directivos que imponen nue- } \\
\text { vos principios que van en contra de los } \\
\text { valores tenían establecidos. }\end{array}$ & $\begin{array}{l}\text { Pérdida de participación en el } \\
\text { mercado donde venden los pro- } \\
\text { ductos. } \\
\text { Desprestigio por parte de los } \\
\text { clientes que toman la decisión } \\
\text { de no comprarle sus productos } \\
\text { porque están disgustados. }\end{array}$ \\
\hline
\end{tabular}




\begin{tabular}{|c|c|c|c|}
\hline Riesgo & ¿Qué puede suceder? & ¿Por qué puede suceder? & ¿Cuáles son las consecuencias? \\
\hline $\begin{array}{l}\text { Mal estado de la mate- } \\
\text { ria prima. }\end{array}$ & $\begin{array}{l}\text { La materia prima se encuentra } \\
\text { con varios defectos, ya sea por } \\
\text { sus características o que no } \\
\text { es la que corresponde para los } \\
\text { productos a desarrollar. } \\
\text { Si se implementa una materia } \\
\text { prima de muy mala calidad, el } \\
\text { resultado final es un producto } \\
\text { muy deficiente para los clientes, } \\
\text { así se cuente con procedimien- } \\
\text { tos y tecnologías muy técnicas. }\end{array}$ & $\begin{array}{l}\text { El proveedor de materia prima en condi- } \\
\text { ciones inadecuadas. } \\
\text { Mal manejo de la materia prima que } \\
\text { esta almacenada. } \\
\text { La empresa decide comprar materia } \\
\text { prima en mal estado por reducir costos. } \\
\text { El proveedor no ofrezca un buen ser- } \\
\text { vicio al cliente en caso de encontrar } \\
\text { defectos en el pedido. }\end{array}$ & $\begin{array}{l}\text { Productos que no cumplen los } \\
\text { requerimientos de los clientes. } \\
\text { - Pérdida de la participación del } \\
\text { mercado. } \\
\text { - Sobreproducción y re-procesos } \\
\text { generando sobrecostos. } \\
\text { - Generación de antivalores con } \\
\text { respecto a la gestión de calidad. }\end{array}$ \\
\hline $\begin{array}{l}\text { Fallas en los procedi- } \\
\text { mientos operacionales } \\
\text { de las máquinas. }\end{array}$ & $\begin{array}{l}\text { La maquinaria que posee la em- } \\
\text { presa que funciona con un flujo } \\
\text { continuo, presente una falla que } \\
\text { hace parar todo el proceso. Esto } \\
\text { ocurre de manera inesperada y } \\
\text { no se conoce la causa de falla la } \\
\text { cual evita que pueda funcionar. }\end{array}$ & $\begin{array}{l}\text { - No exista una programación para un } \\
\text { mantenimiento preventivo que permita } \\
\text { limpiar, lubricar y reparar los posibles } \\
\text { daños por su utilización. } \\
\text { - Falta de capacitación al personal que } \\
\text { no conoce los procedimientos para un } \\
\text { adecuado mantenimiento. } \\
\text { - Falta de especificaciones de cuidados y } \\
\text { control por parte del proveedor. } \\
\text { La empresa solamente hace manteni- } \\
\text { mientos correctivos. }\end{array}$ & $\begin{array}{l}\text { - Paradas del sistema, generando } \\
\text { tiempos muertos, los cuales no } \\
\text { se pueden recuperar. } \\
\text { - Sobrecostos por paros ya que a } \\
\text { los empleados se les paga ese } \\
\text { día laboral. } \\
\text { - Incumplimiento en el tiempo de } \\
\text { entrega. } \\
\text { - Daños parciales y totales a las } \\
\text { maquinas lo que reduce su ciclo } \\
\text { de vida. } \\
\text { Dificultad en el retorno de la } \\
\text { inversión de las maquinas. }\end{array}$ \\
\hline
\end{tabular}


minó a través de dos variables una cualitativa y a otra de tipo monetaria. Ambas determinan el valor a evaluar con respecto a la consecuencia del riesgo.

Tabla 6. Cálculo de la probabilidad

\begin{tabular}{|l|c|}
\hline \multicolumn{1}{|c|}{ Categoría } & Valoración \\
\hline Raro (puede ocurrir casi nunca) & 1 \\
\hline Improbable (puede ocurrir ocasionalmente) & 2 \\
\hline Posible (puede ocurrir en cualquier momento futuro) & 3 \\
\hline Probable (probablemente va a ocurrir) & 4 \\
\hline Muy probable (ocurre en la mayoría de circunstancias) & 5 \\
\hline
\end{tabular}

Fuente: Elaborado y modificado por el autor basado en COLOMBIA COMPRA EFICIENTE. 2012.

Tabla 7. Consecuencia del riesgo

\begin{tabular}{|c|l|l|l|l|l|}
\hline \multirow{4}{*}{$\begin{array}{c}\text { Calificación } \\
\text { cualitativa }\end{array}$} & $\begin{array}{l}\text { Afecta el desa- } \\
\text { rrollo de los obje- } \\
\text { tivos de manera } \\
\text { trivial }\end{array}$ & $\begin{array}{l}\text { Dificulta el } \\
\text { desarrollo de } \\
\text { los objetivos de } \\
\text { manera baja }\end{array}$ & $\begin{array}{l}\text { Afecta el desa- } \\
\text { rrollo del obje- } \\
\text { tivo de manera } \\
\text { media }\end{array}$ & $\begin{array}{l}\text { Obstruye la } \\
\text { ejecución de } \\
\text { objetivo signi- } \\
\text { ficativamente } \\
\text { dificultando su } \\
\text { proceso }\end{array}$ & $\begin{array}{l}\text { Altera la ejecu- } \\
\text { ción del objetivo } \\
\text { de manera grave } \\
\text { dificultado su } \\
\text { avance }\end{array}$ \\
\cline { 2 - 7 } & $\begin{array}{l}\text { Los sobrecostos } \\
\text { no presentan } \\
\text { molificación } \\
\text { mós del 1\% del } \\
\text { valor invertido en } \\
\text { la producción }\end{array}$ & $\begin{array}{l}\text { Los sobrecostos } \\
\text { no presentan } \\
\text { más del 5\% del } \\
\text { valor invertido en } \\
\text { la producción }\end{array}$ & $\begin{array}{l}\text { Genera un } \\
\text { impacto sobre } \\
\text { el valor invertido } \\
\text { en la producción } \\
\text { entre el 5\% y el } \\
15 \%\end{array}$ & $\begin{array}{l}\text { Incrementa el } \\
\text { valor invertido } \\
\text { en la producción } \\
\text { entre el 15\% y el } \\
30 \%\end{array}$ & $\begin{array}{l}\text { Impacto sobre el } \\
\text { valor invertido en } \\
\text { la producción en } \\
\text { más del 30\%. }\end{array}$ \\
\cline { 2 - 7 } & Insignificante & Menor & Moderado & Mayor & Catastrófico \\
\cline { 2 - 7 } & 1 & 2 & 3 & 4 & 5 \\
\hline
\end{tabular}

Fuente: Elaborado y modificado por el autor basado en COLOMBIA COMPRA EFICIENTE. 2012.

Tabla 8. Categoría del riesgo

\begin{tabular}{|l|l|}
\multicolumn{1}{|c|}{ Valoración del riesgo } & \multicolumn{1}{c|}{ Categoría } \\
\hline $8,9,10$ & Riego extremo \\
\hline 6,7 & Riesgo alto \\
\hline 5 & Riesgo medio \\
\hline $2,3,4$ & Riesgo bajo \\
\hline
\end{tabular}

Fuente: COLOMBIA COMPRA EFICIENTE. 2012. 
LIINEA DE INVESTIGACIÓN: DESARROLLO MIPYMES

\subsubsection{Evaluación del riesgo}

Una vez comprendiendo las herramientas que determinan la consecuencia y probabilidad para los diferentes riesgos, en la Tabla 10, se calificó cada riesgo de acuerdo a su respectivo puntaje en las variables anteriores. El puntaje obtenido determina la categoría del riesgo tanto por el color y por el valor cuantitativo (ver Tabla 8)

\section{Tabla 9. Valoración del riesgo}

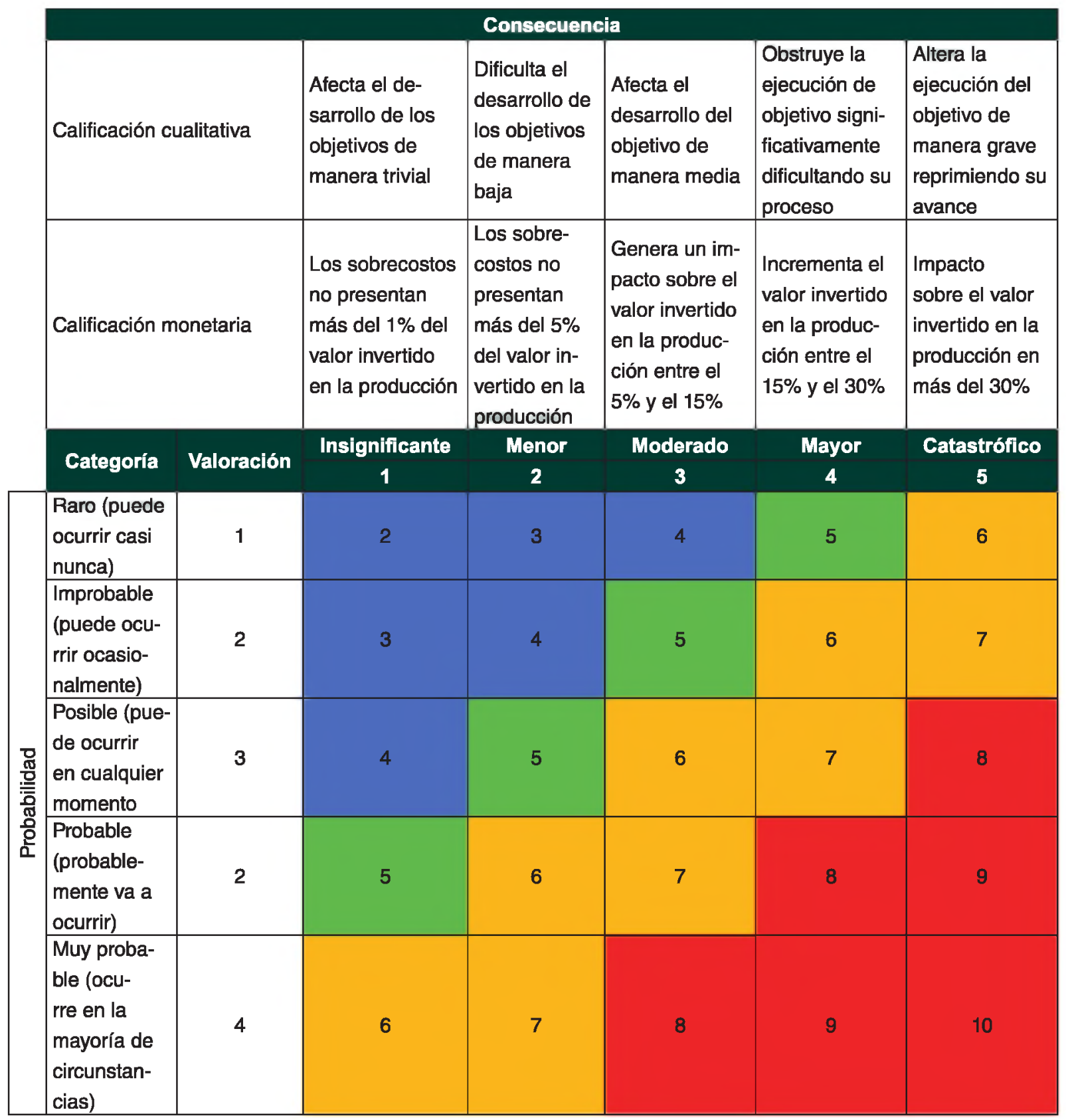

Fuente: Elaborado y modificado por el autor basado en COLOMBIA COMPRA EFICIENTE. 2012. 
Tabla 10. Evaluación de los riesgos

\begin{tabular}{|c|c|c|c|c|}
\hline Riesgo & Probabilidad & Consecuencia & $\begin{array}{l}\text { Valoración } \\
\text { riesgo }\end{array}$ & Categoría \\
\hline $\begin{array}{l}\text { Finalización del ciclo de vida } \\
\text { del producto. }\end{array}$ & 3 & 5 & 8 & Riesgo extremo \\
\hline $\begin{array}{l}\text { Entregar despachos después } \\
\text { del tiempo acordado. }\end{array}$ & 2 & 4 & 6 & Riesgo alto \\
\hline $\begin{array}{l}\text { Defectos en los productos ge- } \\
\text { nerados en su transporte. }\end{array}$ & 2 & 3 & 5 & Riesgo medio \\
\hline $\begin{array}{l}\text { Cantidad errónea de productos } \\
\text { entregados. }\end{array}$ & 2 & 2 & 4 & Riesgo bajo \\
\hline $\begin{array}{l}\text { Pérdida de la imagen corpora- } \\
\text { tiva. }\end{array}$ & 1 & 4 & 5 & Riesgo medio \\
\hline Mal estado de la materia prima. & 2 & 5 & 7 & Riesgo alto \\
\hline $\begin{array}{l}\text { Fallas en los procedimientos } \\
\text { operacionales de las máquinas. }\end{array}$ & 4 & 5 & 9 & Riesgo extremo \\
\hline Desmotivación laboral. & 3 & 3 & 6 & Riesgo alto \\
\hline
\end{tabular}

Fuente: El autor.

El riesgo que mayor puntaje tuvo fue Fallas en los procedimientos de operaciones de las máquinas, ahora se debe de determinar que factor de evaluación se puede reducir.

\section{TRATAMIENTO DEL RIESGO Y MONITOREO O REVISIÓN}

La gestión de riesgos finalizó con la aplicación de los controles preventivos asociados a la identificación del riesgo que mayor impacto afectó el logro de los objetivos empresariales. Según ELASTOPLAST Ltda., la consecuencia por el paro de las máquinas es casi imposible eliminarla, pero los efectos cuando ocurre se ven reflejados en sobrecostos que se pueden salir de un presupuesto. Por esta razón el factor de evaluación que se debe reducir es la probabilidad de ocurrencia.

De acuerdo con las causas la empresa desarrolló las siguientes estrategias que le permitió reducir la probabilidad del riesgo.
Falta de una programación de un mantenimiento preventivo que permita limpiar, lubricar y reparar los posibles daños por su utilización: crear un plan de prevención donde se establezcan fechas especificas para realizar los mantenimientos, limpieza y lubricación.

Falta de capacitación al personal que no conoce los procedimientos para un adecuado mantenimiento: la empresa se capacita a través de una empresa que ofrece el servicio por tercerización, y en algunos casos ellos pueden hacer el mantenimiento.

La empresa solamente hace mantenimientos correctivos: a mediano y largo plazo eliminarlos, y que no se reporte ningún incidente con respecto a este tipo de mantenimiento.

\section{CONCLUSIONES}

Los valores organizacionales, son una base fundamental en la implementación de la ges- 
LÍNEA DE INVESTIGACIÓN: DESARROLLO MIPYMES

tión de riesgos, ya que la alta gerencia debe fomentar los valores que facilitan que los trabajadores estén enfocados en el logro de los objetivos empresariales. Los "antivalores", son un riesgo que las empresas pueden tratar y que este puede generar también graves consecuencias.

Los beneficios esperados al implementar la gestión de riesgos se logran a partir del tratamiento, monitoreo y revisión de los procesos realizados para la gestión de riesgos. Se deben de ir desarrollando de izquierda a derecha como se identifica en el Diagrama 2.

A medida que los controles aplicados al tratamiento del riesgo generan efecto en la prevención del mismo, la gestión de riesgo genera valor en la organización ya que al disminuir la probabilidad de que las máquinas fallen,

Una vez se reduce el riesgo de mayor impacto se puede proceder a realizar el tratamiento para el siguiente riesgo que presenta un valor de tipo riesgo extremo, pero los controles deben de estar patrocinados por la alta gerencia ya que esta debe de asignar los recursos necesarios para su funcionamiento.

Los riesgos que identifica la gestión de riesgo se asocian con variables que la empresa no puede eliminar ni cambiar, pero si no se consideran asertivamente, se pueden llegar a tomar decisiones con un alto grado de incertidumbre que puede afectar considerablemente el logro de los objetivos.

\section{REFERENCIAS}

Bravo, Oscar; Sánchez, Marleny;. (2012). "Gestión Integral de Riesgos". Bogotá D.C.

Cámara de Comercio de Bogotá; "Negocios Responsables y Seguros"; Embajada Británica Bogotá; Oficina de las Naciones Unidas "Contra la Droga y el Delito". (Mayo de 2012). Modelo de Gestión del Riesgo de LA/FT para el sector real. Obtenido de <http:// www.supersociedades.gov.co/ inspeccion-vigilancia-y-control/ prevencion-riesgo-lavado-deactivos/modelo-de-gestiondel-riesgo-de-la-ft/Documents/ MODELO\%20NEGOCIOS\%20 RESPONSABLES $\% 20 Y \% 20$ SEGUROS.pdf

Colombia Compra Eficiente. (Mayo de 2012). Colombia Compra Eficiente. Obtenido de <http://www.colombiacompra.gov.co/sites/default/files/ manuales/cce_manual_riesgo_ web.pdf>

DANE. (Abril de 2013). Obtenido de <https://www.dane. gov.co/index.php/cuentas-economicas/cuentas-trimestrales>

Gutiérrez, M. (2009). Administrar para la calidad, conceptos administrativos del control total de calidad. México D.F: Limusa.

ICONTEC. (2009). Norna NTC ISO 31000. Gestión del Riesgo. Principios y Directrices. Bogotá: ICONTEC.

Jiménez, J. C. (2010). El valor de los valores en las organizaciones. Carácas. 